\title{
Induction of ICAM 1 expression on bladder tumours by BCG immunotherapy
}

\author{
A M Jackson, A B Alexandroff, M McIntyre, K Esuvaranathan, K James, G D Chisholm
}

\begin{abstract}
Aims-To determine the expression of intercellular adhesion molecule 1 and 2 (ICAM 1 and 2) in transitional cell carcinoma cells before and after immunotherapy with Calmette-Guérin bacillus (BCG).

Methods-Frozen sections from 22 untreated bladder carcinomas were immunohistochemically examined with monoclonal antibodies to ICAM 1 and 2. Urinary cytospin slides were made for six patients for each of the six clinical instillations which constitute a therapeutic course. These slides were also stained for ICAM 1 and for leucocyte function associated antigen 1 (LFA 1).

Results-Bladder cancer cells did not essentially express either ICAM 1 or 2, but cells in the stromal areas surrounding tumour expressed both these antigens. After repeated instillations of BCG organisms ICAM 1 positive normal and neoplastic epithelial cells were observed in the urine. Cells obtained from the first three instillations expressed lower densities of ICAM 1 than those from the later instillations. Many neutrophils expressing LFA-1 and some lymphocytes were also noted in the cytospin slides and some of these were conjugated to tumour cells expressing ICAM 1. Six months after treatment a single maintenance dose of BCG induced ICAM 1 expression.

Conclusion-Untreated superficial bladder carcinoma cells do not express ICAM 1 or 2 , but these important immunological molecules were expressed in the stromal areas of tissue. Importantly, neoplastic cells in the urine expressed ICAM 1 after immunotherapy. This molecule can render bladder tumour cells vulnerable to non-antigen specific cytotoxicity mediated by activated lymphocytes.
\end{abstract}

$(\Im$ Clin Pathol 1994;47:309-312)

Department of Surgery, University of Edinburgh

A M Jackson

A B Alexandroff

M McIntyre

K Esuvaranathan K James

G D Chisholm

Correspondence to: Dr A M Jackson, Department of Surger (WGH), University of Edinburgh Medical School, Teviot Place, Edinburgh EH8 9AG

Accepted for publication 27 October 1993 of immunologically active cells into the der wall $^{3}$ and the secretion in the urine of interleukin 1 (IL 1), IL 2, tumour necrosis factor $a(\mathrm{TNF} a)$ and interferon $\gamma($ IFN $\gamma) .^{4-6}$ Furthermore, the neo-expression of mixed histocompatibility complex (MHC) class II molecules by the tumour cells has been reported, ${ }^{7}$ though the in vivo relevance of this is unknown.

In vitro we have shown that therapeutically achievable urine concentrations of IFN $\gamma$ can induce and increase the expression of both MHC class II and intercellular adhesion molecule 1 (ICAM 1, CD54) on bladder tumour cell lines. ${ }^{8-11}$ ICAM 1 is a cytokine inducible adhesion molecule (molecular weight 95 kilodaltons) and is one of three known counterreceptors for the major $\beta 2$ integrin, leucocyte function-associated antigen 1 (LFA 1, CD11a/CD18). ${ }^{12-14}$ The interaction between ICAM and LFA 1 is important for several basic immunological processes, including antigen presentation to $T$ lymphocytes and the extravasation of leucocytes through activated vascular endothelium. ${ }^{15}$ LFA 1 dependent functions are tightly regulated to prevent erroneous conjugation with other cells. ${ }^{16-18}$ Our earlier studies have implicated ICAM 1 in the interaction between activated lymphocytes and bladder tumour cells. ${ }^{9}$ Following such LFA 1/ICAM dependent conjugation events, the activated effector cell was able to mediate the eventual destruction of tumour cells. ${ }^{19}$ In these studies antibodies to either ICAM 1 or LFA 1 negated the conjugation of effector cells with tumour targets and the subsequent delivery of "lethal-hit". 920 If such conjugation were to occur in vivo, after instillation of BCG organisms into the bladder then ICAM 1 expression on the tumour cell may well be required. We therefore investigated the constitutive expression of ICAM 1 and ICAM 2 on bladder tumour biopsy specimens from untreated patients and on urinary cytospin preparations obtained after intravesical BCG treatment of this malignancy.

\section{Methods}

Biopsy material from patients with histologically confirmed transitional cell carcinoma of the bladder was taken and stored in liquid nitrogen for further processing. Patients were selected to undergo immunotherapy with intravesical instillations of BCG. Non-invasive urinary cytospin specimens were obtained from these patients during each week of, and after, treatment. Patients were followed up with check cytoscopies, biopsies, and urine cytology performed at three monthly intervals. After the first six months their initial response to treatment was assessed and correlated with immunohistochemical findings. Patients who were free of disease at this point received a single dose of BCG as maintenance treat- 
ment. Patients whose disease had relapsed received cysto-urethectomy.

Evans (Glaxo) high strength percutaneous BCG in lyophilised form was used. Twenty ampoules containing a total of 1-5 $\times 10^{9} \mathrm{cfu}$ was mixed with $50 \mathrm{ml}$ of physiological saline and instilled into the bladder through a urinary catheter which was subsequently removed. The patients retained the suspension for two hours, during which they were asked to lay on their beds turning from supine to lateral to prone positions at 15 minute intervals in order to expose all surfaces of the bladder to the suspension. Instillations were repeated weekly for six weeks.

\section{PREPARATION OF FROZEN SECTIONS AND} URINARY CYTOSPIN SLIDES

Frozen sections were cut to $5 \mu \mathrm{m}$ thickness at $-25^{\circ} \mathrm{C}$ and mounted on to poly-L-lysine coated glass slides for staining. Cytospins were prepared as follows: freshly voided midstream urine was centrifuged at $1500 \mathrm{rpm}$ for five minutes. The pellet was resuspended in a few millilitres of serum-enriched RPMI 1640 (Gibco, Paisley, UK). Using a cytocentrifuge, $200 \mu \mathrm{l}$ of cell suspension was spun at $700 \mathrm{rpm}$ for eight minutes against poly-L-lysine coated glass slides. All specimens were air dried for 20 minutes, then fixed in acetone for a further 20 minutes, and again air dried before wrapping in aluminium foil and storing at $-20^{\circ} \mathrm{C}$.

\section{MONOCLONAL AND POLYCLONAL ANTIBODIES} Murine monoclonal antibodies to ICAM 1 (RR1/1) and ICAM 2 (CBRIC2) were the generous gift of Dr TA Springer (Centre for Blood Research, Boston, USA). Biotinylated rabbit antibodies to mouse immunoglobulins were obtained from Dako, UK. Horseradish peroxidase and alkaline phosphatase conjugated rabbit antibodies to mouse immunoglobulins were also obtained from Dako.

\section{IMMUNOHISTOCHEMICAL STAINING}

Slides were thawed for 20 minutes before rehydration in $0.2 \mathrm{M}$ TRIS-buffered saline, pH8.6 (TBS), for five minutes. Optimal concentrations of primary antibody were then applied to the specimens which were incubated at $18^{\circ} \mathrm{C}$ for one hour. Following thorough but gentle washing in TBS, specimens were incubated with either biotinylated anti-mouse immunoglobulins or horseradish peroxidase (HRP) conjugated anti-mouse immunoglobulins for a further 30 minutes.

Table 1 Essential expression of ICAM 1 and ICAM 2 by bladder tumours

\begin{tabular}{|c|c|c|c|c|c|c|c|}
\hline \multirow[b]{3}{*}{ Stage } & \multirow[b]{3}{*}{ Mean grade } & \multicolumn{6}{|c|}{ Number of patients expressing: } \\
\hline & & \multicolumn{3}{|c|}{ ICAM 1} & \multicolumn{3}{|c|}{ ICAM 2} \\
\hline & & + & $+1-$ & - & + & $+1-$ & - \\
\hline $\begin{array}{l}\text { Ta } \\
\text { T1 } \\
\text { T2 } \\
\text { T3+ }\end{array}$ & $\begin{array}{l}1.6 \\
2.4 \\
3.0 \\
2.9\end{array}$ & $\begin{array}{l}0 \\
0 \\
0 \\
0\end{array}$ & $\begin{array}{l}0 \\
0 \\
1 \\
1\end{array}$ & $\begin{array}{l}4 \\
6 \\
4 \\
6\end{array}$ & $\begin{array}{l}0 \\
0 \\
0 \\
0\end{array}$ & $\begin{array}{l}0 \\
0 \\
0 \\
0\end{array}$ & $\begin{array}{l}4 \\
6 \\
5 \\
7\end{array}$ \\
\hline
\end{tabular}

Sequential frozen sections obtained from routine biopsy samples were immunohistochemically stained for ICAM 1 and ICAM 2 antigens. The results are ordered with respect to clinical stage of the disease which reflects the degree of invasiveness. Where more than $50 \%$ of epithelial cells expressed antigen, cells were scored $(+)$; between $10-50 \%$ positive cells $(+/-)$; and less than $10 \%(-)$.
After a further wash in TBS, biotinylated immunoglobulins were detected by incubating with alkaline-phosphatase conjugated strepavidin (Dako) for 20 minutes. After further washing, Dako Fast Red substrate was then added for 20 minutes. Slides incubated with HRP-conjugated immunoglobulins were flooded with substrate solution $(3 \mathrm{mg}$ diaminobenzidine solution containing $0 \cdot 1 \%$ hydrogen peroxide) and incubated for 10 minutes. After washing, all slides were lightly counterstained using Mayer's haematoxylin for two minutes and mounted in aqueous neutral glycerine jelly. In negative controls the primary antibody was omitted. Expression was evaluated as follows: negative $(-)$ less than $10 \%$ cells positive; weak $(+/-)$ less than $50 \%$ cells positive; and strong $(+)$ more than $50 \%$ cells expressing ICAM 1 . At least 200 cells were counted for sections; on urinary cytospins a minimum of 100 cells were counted for each sample.

Student's $t$-test was applied for the percentages of cells expressing ICAM 1 in the first and last round of treatment. $P$ values were obtained from standard tables.

\section{Results}

ICAM 1 EXPRESSION IN UNTREATED BLADDER TUMOURS

We have shown that bladder cancer cell lines essentially express either ICAM 1 or ICAM 2 molecules on their surface. ${ }^{910}$ Furthermore, after treatment with the cytokines identified in patients' urine samples, bladder tumour cells in vitro expressed increased concentrations of ICAM 1, but not ICAM 2. These molecules seem to be important for the recognition of tumour cells by activated lymphocytes and may be involved in a successful host response to BCG immunotherapy. In this study we investigated the expression of these antigens in bladder tumours themselves.

Most tumour cells of a variety of histopathological grades (22 patients in total) did not express either ICAM 1 or ICAM 2 (table 1, fig 1). No tumours scored strongly positive $(+)$ for ICAM 1 , and only two of 22 scored weakly positive $(+/-)$, the remainder being negative. None of the tumour cells in the sections examined expressed ICAM 2 . Interestingly, expression of ICAM 1 and, to a lesser extent, ICAM-2, was noted in the stromal regions surrounding areas of carcinoma cells. Stromal expression of these antigens was noted for most patient sections-17 of $22-$ and expression of ICAM 1 was also confirmed on vascular endothelium (not shown).

\section{ICAM 1 POSITIVE TUMOUR CELLS IN URINE} AFTER BCG TREATMENT

Expression of ICAM 1 was studied on tumour cells in urinary cytospin slides obtained from each of the six instillations from six patients undergoing a course of BCG immunotherapy. The neoplastic status of suspected tumour cells was confirmed by a consultant histopathologist. In cells obtained 12 hours after the first two instillations of BCG, ICAM 1 expression was either absent or weak. After 
Table 2 Expression of ICAM 1 on epithelial cells shed into patients' urine after instillations of BCG organisms

\begin{tabular}{lllllll}
\hline Case No & 1st & 2nd & 3rd & 4 th & 5 th & 6 th \\
\hline 1 & - & - & - & + & + & + \\
2 & $+1-$ & - & + & + & + & + \\
3 & - & - & - & + & + & + \\
4 & - & $+1-$ & + & + & + & + \\
5 & - & & + & + & + & + \\
6 & & & - & - & + & + \\
\hline
\end{tabular}

Urinary cytospins were made from a fresh urine sample obtained 12 hours after each of six instillations of BGC and then immunohistochemically stained for ICAM 1 expression. Where more than $50 \%$ of epithelial cells expressed antigen, cells were scored $(+)$; between $10-50 \%$ positive cells $(+l-)$; and less than $10 \%(-)$

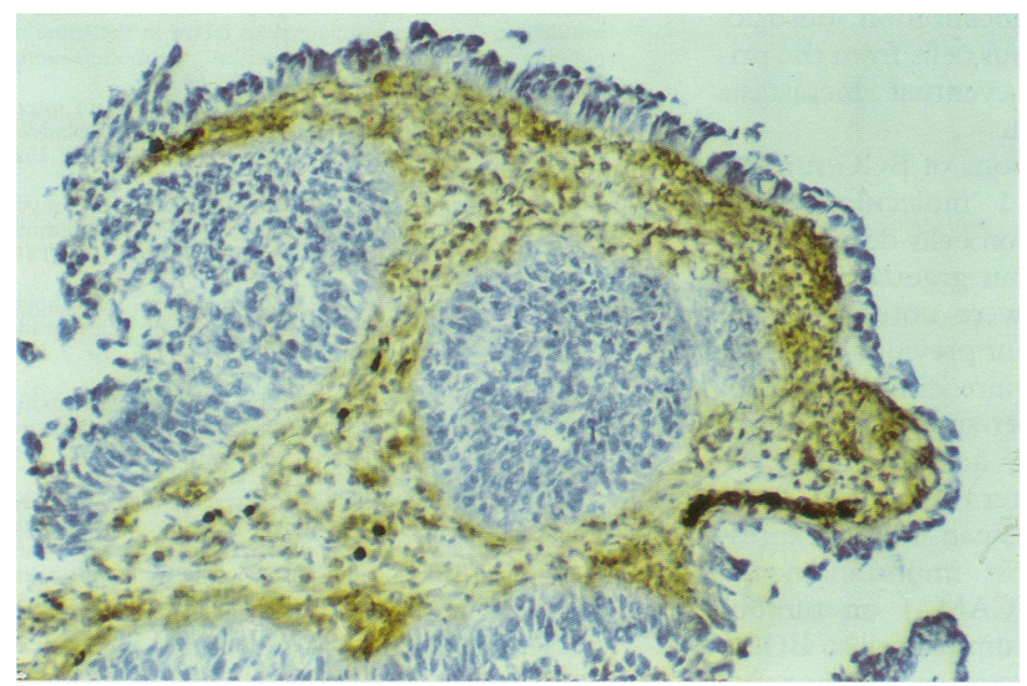

Figure 1 Immunohistochemistry of ICAM 1 on a frozen section obtained from an untreated superficial bladder tumour. ICAM 1 was detected using monoclonal antibodies and a secondary polyclonal antibody conjugated to horseradish peroxidase. The substrate used was $D A B$, which yields a brown stain. Note: ICAM 1 expression is localised to the stromal areas. Tumour cells (blue) did not express ICAM 1. ICAM 2 expression was also absent on tumour cells and weaker in stromal regions (not shown).

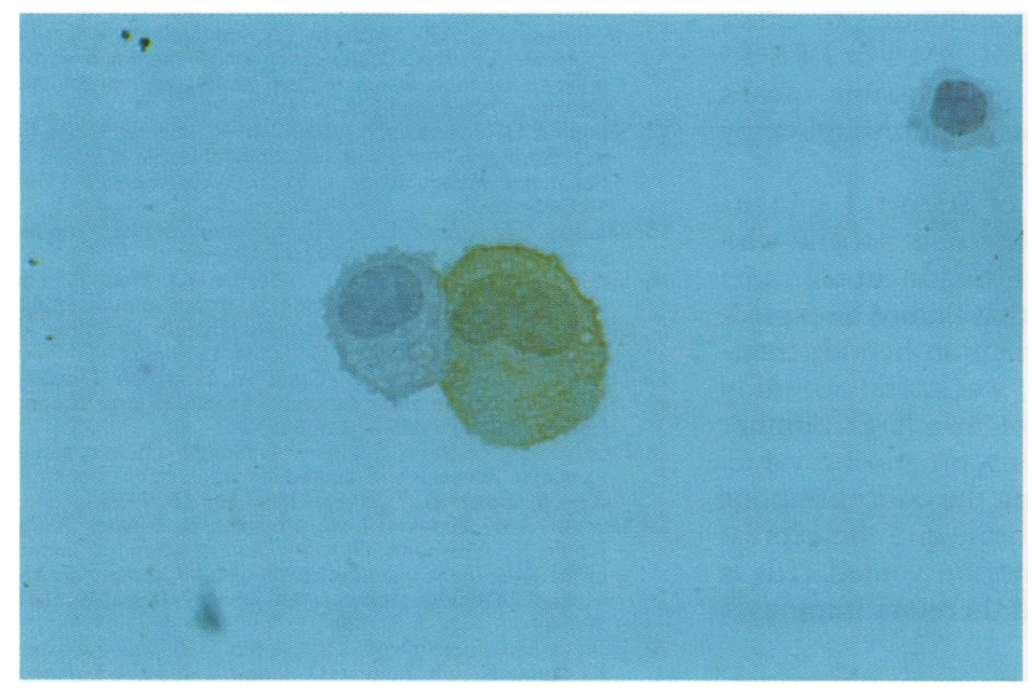

Figure 2 Detection of ICAM 1 expression on urinary cytospins obtained after treatment with repeated instillations of BCG. Shown are two epithelial cells, one of which expressed ICAM 1 (brown).

Table 3 Expression of ICAM 1 on epithelial cells shed into patients' urine after maintenance treatment

\begin{tabular}{lllllllll}
\hline Case No & 2 & 5 & 6 & 7 & 8 & 9 & 10 & 11 \\
lst instillation & $+/-$ & - & & & - & - & & - \\
Maintenance & + & $+/-$ & + & + & + & - & + & +
\end{tabular}

Urinary cytospins were made from a fresh urine sample obtained 12 hours after treatment and then immunohistochemically stained for ICAM 1 expression. Where more than $50 \%$ of epithelia cells expressed antigen, cells were scored $(+)$; between $10-50 \%$ positive cells $(+/-)$; and less than $10 \%(-)$. the third instillation three patients had high levels of ICAM 1 expression. Malignant and normal epithelial cells obtained from the later instillations, however, ${ }^{4-6}$ from all patients showed that more than $50 \%$ of these cell types expressed ICAM 1 (table 2, fig 2). A significance value of $p<0.001$ was obtained when the difference in ICAM 1 expression on cells obtained after one instillation of BCG and six instillations were compared.

Tumour cells were not stained for ICAM 2 expression after BCG treatment as ICAM 2 is not essentially expressed and is known not to be a cytokine inducible gene.

\section{MAINTENANCE TREATMENT}

The expression of ICAM 1 was maintained for at least six months after the final instillation of BCG. After six months patients who had undergone a complete response to immunotherapy (as judged by cystoscopic examination) were given a single instillation of BCG organisms, termed "maintenance." The expression of ICAM 1 on normal epithelial cells shed into the urine was also studied using immunohistochemistry. Unlike the first course of treatment, where expression of ICAM 1 was initially absent, over $50 \%$ of cells obtained after the maintenance course were positive for ICAM 1 expression. The data showing first response to BCG and "maintenance" are summarised in table 3.

\section{Discussion}

Intravesical BCG treatment for superficial bladder cancer is the most successful form of immunotherapy for any solid human malignancy. ${ }^{1}$ The mechanisms of action, however, remain largely undetermined. After repeated BCG instillation high concentrations of certain cytokines are readily detected in the urine; patients receiving intravesical chemotherapy do not secrete cytokines. ${ }^{4-6}$ These cytokines are probably involved in the antitumour response of the host.

In vitro, when bladder cancer cells are treated with the recombinant versions of the cytokines detected in the urine, they respond in a variety of ways. They express MHC class II and ICAM 1 antigens and their growth characteristics are altered..$^{81021}$ In vivo we have observed the expression of MHC class II molecules after repeated instillations of BCG. ${ }^{7}$ This study was designed to investigate the expression of ICAM 1 by tumour cells in patients during and after BCG treatment.

Transitional cell carcinomas of the bladder did not essentially express either ICAM 1 or ICAM 2 molecules. It might be advantageous not to express ICAM and thus evade tumour detection by infiltrating leucocytes. Our in vitro conjugation and cytotoxicity studies have done much to confirm the importance of ICAM 1 expression in increased vulnerability to killing by activated lymphocytes. ${ }^{919} 20$ For other tumours, however, a correlation has been made between ICAM 1 expression and increased propensity for metastasis. ${ }^{22}$ In this study metastatic tumour expressed higher ICAM 1 concentrations than the primary 
lesion. The means by which increased ICAM 1 expression might lead to a poorer prognosis remain unclear, however: ICAM-1 can be shed from cells and may block infiltrating leucocytes. ${ }^{23}$ One study correlated increased concentrations of circulating ICAM 1 with the presence of metastatic cancer; patients with a primary lesion alone had significantly lower concentrations. ${ }^{24}$ In this regard we detected soluble forms of ICAM 1 in patients' urine after BCG immunotherapy. ${ }^{25}$ Whether high densities of cell associated ICAM 1 or differences in shedding/membrane turnover are involved in the immunolocalisation, destruction, detachment of tumour cells from the primary lesion, or their eventual metastasis remains to be determined.

After repeated instillations of BCG tumour cells expressed ICAM 1 molecules. Little expression was observed on cells derived after the earlier instillations, but gradual increases in intensity of staining were noted over the later instillations. From our previous studies it seems that this would require stimulation with various cytokines. Whether such cytokines are derived from infiltrating activated lymphocytes remains to be determined, but those cytokines present in urine can induce expression of ICAM 1.11 Most importantly, the induced expression of ICAM 1 on tumour cells is a response of the tumour cell to BCG.

The coupling of tumour cells expressing ICAM 1 with lymphocytes expressing LFA 1 allows "lethal-hits" to be delivered to the tumour cell in vitro. Whether this takes place in vivo requires investigation, but our previous studies have demonstrated increased vulnerability to cytolysis with increasing levels of ICAM 1 expression. Such cytotoxicity is abolished by monoclonal antibodies to either of the adhesion molecules ICAM 1 or LFA $1 .^{9}$ Therefore, this may be one possible means by which BCG activated immunotherapy achieves its clinical success.

The poor expression of ICAM 1 and its rapid disappearance in the one patient who failed to respond to initial treatment warrants further study. It may be that failure to express ICAM 1 after BCG instillation heralds treatment failure. Therefore, we intend to study the expression of ICAM 1 in a large number of patients to evaluate any prognostic value. One advantage in studying the concentrations of cytokines in patients' urine and the expression of adhesion molecules on voided cells is that it is non-invasive and therefore minimises patient distress.

Further experiments are currently underway in an attempt to dissect the role of cellular adhesion molecules in the eradication of tumour cells after immunotherapy with BCG. We are presently investigating the direct effects of BCG on ICAM 1 expression by bladder cancer cells. Investigation of any correlation of ICAM 1 expression with response to clinical treatment is the logical next step and a large study is now under way.

Dr TA Springer generously donated murine monoclonal antibodies to ICAM 1 and ICAM 2, and for this we are grateful.

This ICAM University of Edinburgh Cancer Research Fund and the Royal College of Surgeons Edinburgh. We thank Mr J Black and Mrs

1 Lamm DL, Van der Meijen APM, Morales A, Brosman SA, Catalona WJ, Herr HW, et al. Incidence and treatment of complications of bacillus Calmette-Guérin intravesical therapy in superficial bladder cancer. $₹$ Urol intravesical therapy in

2 Ratliffe TL, Gillen D, Catalona WJ. Incidence and treatment of complications of bacillus Calmette-Guérin intravesical therapy in superficial bladder cancer. $f$ Urol 1987;137:155-8.

3 El Demiry MIM, Smith G, Ritchie AWS, Hargreave TB, Busuttil A, James $\mathrm{K}$, et al. Local immune responses after intravesical BCG treatment for carcinoma in situ. $\mathrm{Br} \mathcal{F}$ Urol 1987;60:543-8.

4 Bohle A, Nowc AJ, Ulmer AJ, Musehold J, Gedes J, Hofster AG, et al. Elevations of cytokines interleukin-1, interleukin-2 and tumour necrosis factor in the urine of patients after intravesical bacillus Calmette-Guérin patients after intravesical bacillus Caln

5 Haaff EO, Catalona WJ, Ratliffe TL. Detection of interleukin-2 in the urine of patients with superficial bladder tumours after treatment with intravesical BCG. $\mathcal{F}$ Urol 1987;136:970-4

6 Prescott S, James K, Hargreave T, Chisholm GD, Smyth JF. Radioimmunoassay detection of interferon-gamma in urine after Evans BCG therapy. $\mathcal{F}$ Urol 1990;144: 1248-51.

7 Prescott S, James K, Busuttil A, Hargreave T, Chisholm GD, Smyth JF. HLA-DR expression by high grade superficial bladder cancer treated by BCG. Br f Urol 1989;63:264-9.

8 Hawkyard SJ, James K, Prescott S, Jackson AM, Ritchie AWS, Smyth JF, et al. The effects of recombinant human interferon-gamma on a panel of hum
cancer cell lines. $\mathcal{f}$ Urol 1991;145:1078-81.

9 Jackson AM, Alexandrov AB, Prescott S, James K, Chisholm GD. Role of adhesion molecules in lymphokine activated killing of bladder cancer cells: Further evidence for a third ligand for leucocyte function associated antigen-1. Immunol 1992;76:286-91.

10 Jackson AM, Alexandrov AB, Prescott S, James $\mathrm{K}$ Chisholm GD. Expression of adhesion molecules by bladder cancer cells: Modulation by interferon-gamma and tumour necrosis factor-alpha. $\mathcal{f}$ Urol 1992;148: and tum $1583-6$.

11 Jackson AM, Alexandrov AB, Prescott S, James $\mathrm{K}$ Chisholm GD. The immunomodulatory effects of urine from patients with superficial bladder cancer receiving intravesical Evans BCG therapy. Cancer Immunol Immunother 1993;36:25-30.

12 Dustin ML, Springer TA. Lymphocyte function-associated antigen (LFA-1) interaction with intercellar adhesion molecule-1 (ICAM-1) is one of at least three mechanisms for lymphocyte adhesion to cultured endothelial cells. 7 Cell Biol 1988;107:321-31.

13 de Fougerolles AR, Springer TA. Intercellular adhesion molecule-3, a third adhesion counter receptor for lymphocyte function associated molecule-1 on resting lymphocyte function associated molecule-

14 Staunton DE, Merluzzi VJ, Rothlein R, Barton R, Marlin SD, Springer TA. A cell adhesion molecule ICAM-1 is
Stanton DE, Merluzzi VJ, Rothlein R, Barton R, Marlin the major surface receptor for rhinociruses. Cell 1989 56:849-53.

15 Mackay CR, Imhof BA. Cell adhesion in the immune system. Immunol Today 1993;14:99-102.

16 Van Seventer GA, Shimizu Y, Horgan KJ, Shaw S. The LFA-1 ligand ICAM-1 provides an important costimulatory signal for $\mathrm{T}$ cell receptor-mediated activation of resting T cells. F Immunol 1990;144:4579-86.

17 Dransfield I, Cabanas C, Craig A, Hogg N. Divalent Dransfield I, Cabanas C, Craig A, Hogg N. Divalent cation regulation of the function of the

18 Dransfield I, Cabanas C, Barrett J, Hogg N. Interaction of leucocyte integrins with ligand is necessary but not sufficient for function. $\mathcal{F}$ Cell Biol 1992;116:1527-35.

19 Jackson AM, Hawkyard SJ, Prescott S, Ritchie AWS James $\mathrm{K}$, Chisholm GD. An investigation of factor influencing the in vitro induction of LAK activity agains a panel of bladder cancer cell lines. $₹$ Urol 1992;147: 207-11.

20 Jackson AM, Alexandroff AB, Lappin MB, Esuvaranathan $K$, James $K$, Chisholm GD. Control of LFA-1 dependent cellular conjugation by divalent cations. Immunol dent cellular con

21 Hawkyard SJ, Jackson AM, James K, Prescott S, Smyth JF, Chisholm GD. The inhibitory effects of interferonJF, Chisholm GD. The inhibitory effects of interferongamma on the growth

22 Natalie P, Nicorta MR, Cavaliere R, Bigotti A. Differential expression of ICAM-1 in primary and metastatic lesions. Cancer Res 1990;50:1271-8.

23 Becker JC, Dummer R, Hartmann AA, Burg G. Shedding of ICAM-1 from human melanoma cell lines induced by IFN $\gamma$ and TNF $a$. $\mathcal{f}$ Immunol 1991;147:4398-401.

24 Tsijiska $M$, Imai $K$, Hirata $H$, Hanzawa Y. Detection of circulating ICAM-1 antigen in patients with malignan disease. Clin Exp Immunol 1991;85:3-8.

25 Jackson AM, Alexandrov AB, Gribben SC, Esuvaranathan $K$, James $K$. Expression and shedding of ICAM-1 in bladder cancer and its immunotherapy. Int 7 Cancer 1993;55:921-5. 